\title{
RELATIONSHIP BETWEEN SPINAL MOBILITY, PHYSICAL PERFORMANCE, PAIN INTENSITY AND FUNCTIONAL DISABILITY IN PATIENTS WITH CHRONIC LOW BACK PAIN
}

\author{
*DO Odebiyi, **SO Kujero and ***TA Lawal \\ Departments of Physiotherapy, College of Medicine, University of Lagos; \\ **Military Hospital, Yaba, Lagos; ***Lagos University Teaching Hospital, Idi-araba Lagos.
}

\author{
Corresponding Author \\ Daniel O Odebiyi \\ e-mail:femiodebiyi@yahoo.com
}

\begin{abstract}
SUMMARY
Background: Physiotherapy clinical assessment has traditionally relied on clinical tests of impairment. These tests correlate poorly with patients' pain and dysfunction, especially in the case of chronic pain. This study was designed to investigate the correlation between some of these tests in patients with chronic low back pain.

Methodology: Twenty-three patients (16 males, 7 females) with chronic low back pain participated in the study. Roland- Morris Disability Questionnaire (R-MDQ), Visual Analogue Scale (VAS) and Physical Performance Battery (PPB) were used to collect data. The spinal range of motion was assessed using a tape measure and a goniometer. Data analysis was done using Pearson Moment Product correlation coefficient and Spearman's Rank.

Results: Statistical analysis showed that there was a significant inverse correlation between repeated trunk flexion and anterior trunk flexion $(r=-0.488, p<0.05)$, extension and 50 -foot walk $(r=-0.462 ; p<0.05)$. There was also a significant correlation between functional disability and 50foot walk $(r=0.456 \mathrm{p}<0.05)$ among all subjects.

Conclusion: Increase in pain may not necessarily result in increase in functional disability, as relevant improvements in pain may lead to almost unnoticeable change in disability clinically. Therefore, self-report of disability by patients may not be adequate in making clinical judgement of their condition without the objective assessment of their physical performance.
\end{abstract}

KEYWORDS: Low back pain, Physical performance, Spinal mobility.

\section{INTRODUCTION}

Low back pain (LBP) is a major health problem worldwide (Frymoyer, 1998). The lifetime prevalence of low back pain is estimated at $60-85 \%$, while the annual prevalence in the general population is ranging from 15-45\% (Burton et al, 1996). The annual incidence of LBP in the general population is estimated between 10\% - 15\% (Andersson, 1999). According to the World Health Organization's International Classification of functioning and Disability (ICF), the impact of LBP on physical performance has been classified into dimensions of impairment, activity (limitation), and participation- restriction (WHO, 2001). Traditional physical tests tend to address impairments (Moffroid et al, 1994), and these impairments; such as postural aberrations, decreased muscle force and range of motion, may not be good indicators of musculoskeletal functions and disability (Mooney, 1987; Waddell et al, 1992).The assessment of pain and disability are necessary components of the management of chronic back pain syndrome, the two main challenges of assessment are to determine the severity of the syndrome and the degree of the response to treatments (Deyo and Weinstein, 2001).

Physiotherapy clinical assessment for individuals living with LBP has traditionally relied on clinical tests of impairment which has been reported to correlate poorly with patients' pain and dysfunction, especially in the case of chronic pain (Waddell, 1987; Turk, 1997). Standardized tests of muscle strength and range of motion in isolation has also been reported to lack sensitivity, specificity, and responsiveness (Nelson and Nester, 1988). Thus complex and expensive isometric and isokinetic devices have been employed to measure muscle strength, range of joint motion and velocity of motion reliably; however, the performance of patients with chronic pain may be erratic, more so because of psychological factors such as fear of injury and low perceived self - efficacy (Newton et al, 1993). Also, it has been reported that specialized testing with these devices does not simulate everyday activities (Simmonds et al, 1998).

The need to develop appropriate tools for measuring mobility and activities of daily living was recently characterized as a priority for research by an international task force on back pain (Abenhaim et al, 2000). Timed tests of activities such as walking, sit-to- stand task, and 
repeated trunk flexion have been examined in patients with back pain and have been shown to have acceptable reliability, to be able to discriminate between people with and without back problems, and also to be sensitive to change over time (Harding et al, 1994; Simmonds et al, 1998; Simmonds, 1999). Several disability scales have been developed for people with LBP, and their importance as measures of treatment outcome in clinical trials has been emphasized (Deyo et al, 1998).

Self - reported measurements of disability have been used as an outcome measure for people with (LBP) (Deyo, 1988). The rationale behind this work was to determine how all these parameters, that is, spinal mobility measures, physical performance tests (which are objective in nature), pain intensity and self-report of functional disability, can be incorporated as a package into physiotherapy assessment and rehabilitation for effective management of patients with chronic low back pain.

This study is aimed at determining the correlation between the range of motion of the spine and physical performance and correlation between pain intensity and functional disability in patients with chronic low back pain.

\section{MATERIALS AND METHODS Subject Selections:}

Twenty three (23) subjects participated in this study, their ages range between 25 - 65 years. They were patients with low back pain (LBP) of not less than 3 months. The subjects were recruited from the out-patient clinic of the Department of Physiotherapy of the Lagos State University Teaching Hospital, Idi-Araba, Lagos and the Physiotherapy Department, National Orthopaedic Hospital, Igbobi, Lagos. Only patients whose LBP radiate to the leg were included in the study. A non-probability sampling technique (consecutive) was employed and only those subjects who met the inclusion criteria were recruited.

\section{Materials:}

The materials used during the course of the study were as follows: Roland and Morris Disability Questionnaire (RMDQ), Visual Analogue Scale (VAS), Simmonds Physical Performance Battery, Seca Height Meter, and Seca weighing scale (weight):

- Roland and Morris Disability Questionnaire: This was used to assess the degree of functional limitation in the subjects. It is a 24- item Questionnaire selected from the sickness impact profile of LBP, the Questionnaire has been used to evaluate outcome in a range of populations, settings and interventions, with a test - retest reliability of 0.84 (Roland and Morris, 1983a).

- Simmonds Physical Performance Battery (SPPB): SPPB involved the performance of tasks that are fundamental component of day-to-day activities that are commonly compromised by LBP (Simmonds et al, 1998). It is a simple and standard battery of performance tests to complement the assessment of patients with LBP. The performance on the battery is generally measured on the basis of how quickly a task can be performed, or how far a patient with LBP can stretch forward (i.e. an indirect measure of spinal load). The equipment needed for these simple clinical performance tests are: stop watch, tape measure and $4.45 \mathrm{~kg}$ weight. The tasks that are usually performed are 1) repeated sitto-stand, 2) repeated trunk flexion, 3) loaded reach, 4) $360^{\circ}$ rollover and 5) Sorensen fatigue test. The clinical utility, reliability and validity of this performance tests has been established (Simmnods et al, 1998).

- Visual analogue scale: This is a $10 \mathrm{~cm}$ callibrated line with 0 (zero) representing no pain and 10 (ten) representing worst pain.The subjects were asked to make a mark/point on the scale that best represent the intensity of pain experienced. The distance between zero and the mark/point was then measured and recorded.

\section{Methods:}

The subjects were required to complete the selfreported questionnaires namely the Roland and Morris Disability Questionnaire and the Visual Analogue Scale.

Spinal mobility: Spinal mobility (i.e. lateral Flexion Spinal Extension and Forward flexion) was assessed with the subjects in erect standing position, and with feet positioned approximately shoulder-width apart. This position was found to stabilize the pelvis and aide in maintenance of balance, and thus helps to increase the consistency of measurements (Simmonds et al, 1998).

- lateral Flexion: The movement was demonstrated to the subjects and the importance of staying in the coronal plane was emphasized. A mark was made $10 \mathrm{~cm}$ above the mid point of an immaginary line (over the vertebral column) joining the two posterior superior iliac spines (PSISs). The goniometer axis was placed over the sacrum midway between the two PSISs. The 
stationary arm of the goniometer was positioned perpendicularly to the floor (i.e. an imaginary line joining the PSISs), and the movable arm was aligned with the spinous process of the seventh cervical vertebra. The subjects were then instructed to bend sideways to the right as far as possible while keeping the knees straight. The stationary arm of the goniometer was held against the subjects' pelvis during the movement rather than being kept perpendicularly to the floor. The range of motion obtained was measured and recorded in degrees after the subjects have achieved maximum movement. The subjects were then asked to return to the upright position, and the same procedure was repeated to measure left lateral flexion.

- Spinal Extension: The movement was demonstrated to the subjects. The subjects were instructed to stand erect with the feet shoulder - width apart. The goniometer axis was placed at the junction of the superior iliac crest and the midaxilary line. The stationary arm of the goniometer was positioned vertical to the floor; the movable arm was aligned with the midaxillary line. The subjects were then instructed to bend backward as far as possible without bending the knees. The range of motion was then measured and recorded in degrees after the subjects have achieved maximum movement.

- Forward flexion: The subjects were asked to return to the upright position after the performance of spinal extention, and the same procedure was repeated but this time the subjects were asked to bend forward as far as possible without bending the knees. The range of motion was then measured and recorded in degrees after the subjects have achieved maximum movement.

- The Physical Performance Tests: The physical performance tests were carried out on the subjects in the following order repeated sit-to-stand, repeated trunk flexion, loaded reach, 50 - foot walk, 5 minute walk, $360^{\circ}$ roll over and Sorensen Fatigue Test.

- Repeated Sit - to - Stand: With the subjects seated in an appropriate chair, they were required to rise to standing position and then return to sitting position as quickly as possible five times. The task was repeated after a brief pause, the average time taken to perform the rask was taken and recorded.

- Repeated Trunk Flexion: The subjects observed a brief pause thereafter they were instructed to assumed an upright position and then bent forward while keeping the knees straight as quickly as possible five times. The task was repeated after a brif pause and the average time taken to perform the task was taken and recorded.

- Loaded Reach: A tape measure was mounted horizontally at the subjects' shoulder height on a wall and the subjects were required to stand with the shoulder directly opposite the tape measure. They were then required to hold a $4.45 \mathrm{~kg}$ weight close to the body and reach forward. The maximum distance reached in $\mathrm{cm}$ on the tape measure was measured and recorded.

- 50 - Feet Walk: The subjects were made to walk twice on a 25 feet walk way as fast as possible. The time taken to complete the task was taken and recorded.

- 5 Minute Walk: The subjects were instructed to walk on a walk way as fast as possible for five minutes. The distance covered was measured and recorded.

- $\quad \mathbf{3 6 0}^{\circ}$ Roll Over: With the subjects in supine on a mat they were instructed to rollover $360^{\circ}$ as fast as possible. After a brief pause, they were instructed to roll over $360^{\circ}$ in he opposite direction. The time taken to complete the task in both direction was taken and recorded

- Sorensen Fatigue Test: Finally, the subjects were required to perform the Sorensen fatigue test in which they were instructed to lay prone on a standard treatment table with the thighs and calves stabilized, and they were then instructed to lift their upper body and hold the position for as long as possible, and the time taken to fatigue was recorded.

\section{Statistical Analysis}

All data were analysed using SPSS statistical software (version 11). Pearson product moment and Spearman's rho correlation were used to determine significant relationship between variables. Mann Whitney $U$ test was also to determine significant difference between variables.

\section{RESULTS}

Table 1: shows the mean values of functional disability and pain intensity of the subjects. Mann Whitney U test showed that there was no statistically significant difference in the RMDQ and VAS scores between the male and female subjects.

Table 2: shows the correlation between spinal mobility and physical performance of the subjects. Statiscal analysis showed that there was an inverse correlation between anterior trunk flexion and repeated trunk flexion $(r=-0.488$; $p<$ $0.05)$; left lateral flexion and repeated sit-to- 
stand ( $\mathrm{r}=-0.424 ; \mathrm{p}<0.05)$; extension and 50foot walk $(\mathrm{r}=-0.462 ; \mathrm{p}<0.05)$. Among the female group, there was a strong inverse correlation between anterior trunk flexion and repeated trunk flexion $(r=-0.792 ; \mathrm{p}<0.05)$. There was also a moderate inverse correlation between right lateral flexion and repeated trunk flexion ( $\mathrm{r}=-0.568, \mathrm{p}<0.05$ ); left lateral flexion and repeated trunk flexion $(r=-0.568, p<0.05)$ among the male group.

Table 3: shows the correlation between pain intensity and functional disability in the subjects. Statiscal analysis showed that there was no correlation between pain intensity and functional disability. There was also a poor correlation which was not statistically significant among the female subjects.

Table 4 shows the correlation between Roland Morris Disability Questionnaire (physical performance tasks) and functional disability (self-report). Statiscal analysis showed that there was a significant correlation $(r=0.456$; $\mathrm{p}<0.05)$ between functional disability and 50 -foot walk among all subjects. However, there was no correlation between functional disability and any of the physical performance tasks between the male and the female subjects.

Table 1: Mean values of functional disability (RMDQ) and pain intensity (VAS).

\begin{tabular}{lllll}
\hline & MALE & \multicolumn{2}{l}{ FEMALE U } & $\mathbf{P}$ - value \\
& $\mathbf{X} \pm($ SD) & $\mathbf{X} \pm($ SD) & & \\
\hline & & & & \\
RMDQ & $8.44 \pm 5.33$ & $9.57 \pm 4.86$ & 19.98 & 0.5 \\
VAS & $4.52 \pm 1.94$ & $6.41 \pm 2.24$ & 12.22 & 0.12 \\
\hline
\end{tabular}

Keys
RMDQ - Roland Morris Disability Questionnaire
VAS $\quad-$ Visual Analogue Scale
SD $\quad$ Standard Deviation

Table 2: Correlation between spinal mobility and physical performance.

\begin{tabular}{llll}
\hline & \multicolumn{3}{c}{ Correlation (r) } \\
& All subjects & Male & Female \\
\hline Anterior trunk flexion \& Repeated sit-to-stand & -0.320 & -0.352 & 0.554 \\
Anterior trunk flexion \& Repeated trunk flexion & $*-0.488$ & -0.453 & $*_{-} 0.792$ \\
Anterior trunk flexion \& Loaded reach & -0.196 & -0.148 & -0.240 \\
Anterior trunk flexion \& 50-foot walk & -0.118 & 0.097 & -0.571 \\
Anterior trunk flexion \& 5-minute walk & 0.037 & 0.108 & 0.117 \\
Anterior trunk flexion \& 360 rollover & -0.315 & -0.197 & -0.643 \\
Anterior trunk flexion \& Sorensen fatigue test & 0.193 & 0.212 & 0.122 \\
Right lateral flexion \& Repeated sit-to-stand & -0.297 & -0.342 & 0.175 \\
Right lateral flexion \& Repeated trunk flexion & $* *-0.554$ & $* 0.568$ & -0.361 \\
Right lateral flexion \& Loaded reach & -0.046 & -0.051 & -0.734 \\
Right lateral flexion \& 50-foot walk & -0.345 & -0.286 & -0.339 \\
Right lateral flexion \& 5-minute walk & 0.298 & 0.306 & -0.246 \\
Right lateral flexion \& 360 ${ }^{\circ}$ rollover & -0.207 & -0.338 & 0.132 \\
Right lateral flexion \& Sorensen fatigue test & 0.024 & 0.442 & -0.416 \\
Left lateral flexion \& Repeated sit-to-stand & $*-0.424$ & -0.415 & -0.325 \\
Left lateral flexion \& Repeated trunk flexion & $* *-0.616$ & $*-0.568$ & -0.624 \\
Left lateral flexion \& Loaded reach & -0.024 & 0.026 & -0.575 \\
Left lateral flexion \& 50-foot walk & -0.390 & -0.188 & -0.654 \\
Left lateral flexion \& 5-minute walk & 0.295 & 0.331 & -0.246 \\
Left lateral flexion \& 360 $0^{\circ}$ rollover & -0.331 & -0.283 & -0.367 \\
Left lateral flexion \& Sorensen fatigue test & 0.143 & 0.428 & -0.200 \\
Extension \& Repeated sit-to-stand & -0.350 & -0.246 & -0.253 \\
Extension \& Repeated trunk flexion & $* *-0.536$ & -0.232 & -0.656 \\
Extension \& Loaded reach & -0.089 & 0.138 & -0.591 \\
Extension \& 50-foot walk & $*-0.462$ & -0.448 & -0.459 \\
Extension \& 5-minute walk & 0.402 & 0.395 & -0.057 \\
Extension \& 360 ${ }^{\circ}$ rollover & -0.257 & -0.158 & -0.315 \\
Extension \& Sorensen fatigue test & -0.134 & 0.313 & -0.444 \\
\hline
\end{tabular}

*Correlation is significant at the 0.05 level (2-tailed). 
Table 3: Correlation between Pain Intensity (Vas Scores) and Roland Morris Disability Questionnaire (Functional Disability).

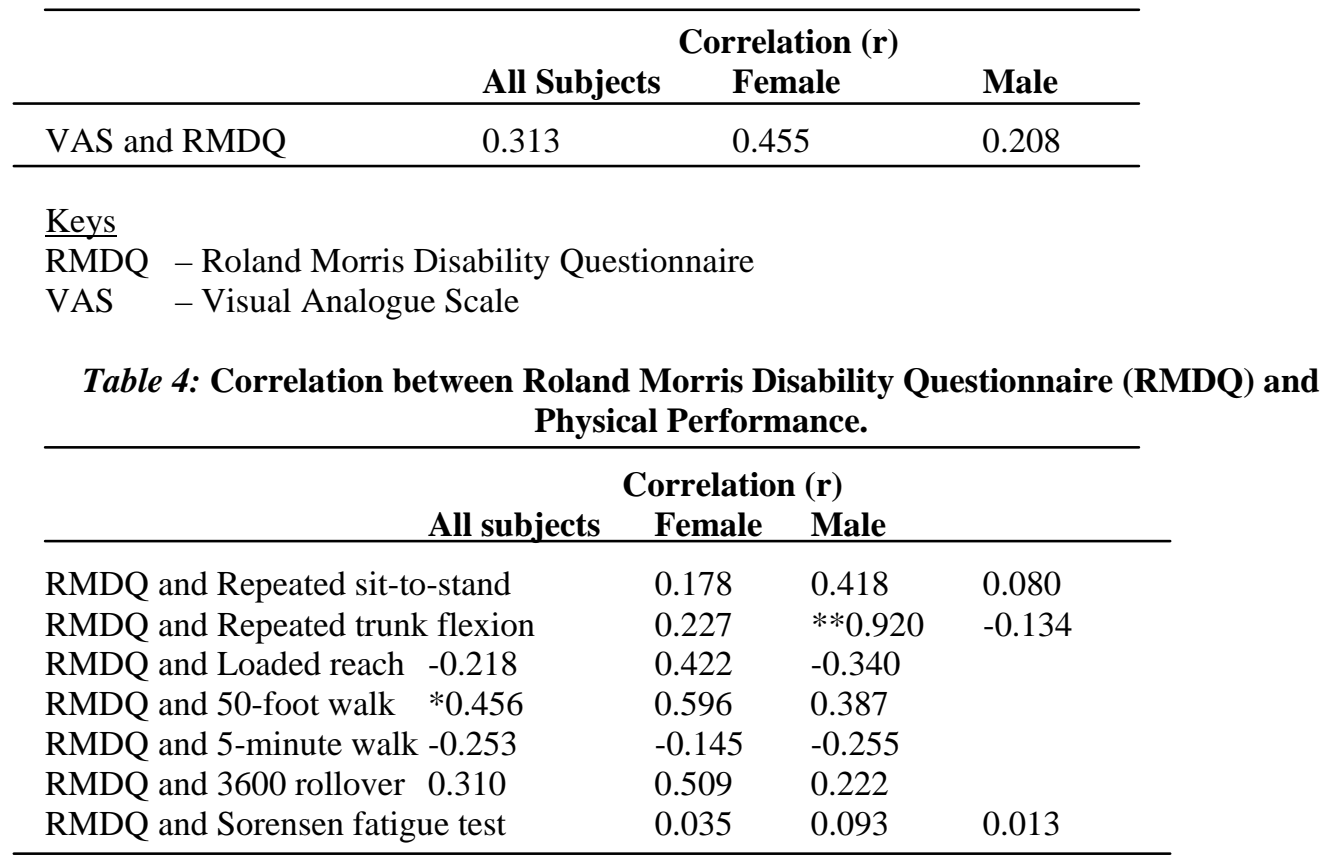

*Correlation is significant at the 0.05 level (2-tailed).

\section{DISCUSSION}

This study was designed to investigate the relationship between spinal mobility, physical performance, pain intensity, and functional disability in patients with chronic low back pain. The result of the study showed that there was a significant negative correlation between pain and all the spinal mobility measures (i.e. anterior trunk flexion, right and left lateral flexion) except back extention. This implies that with increase in the range of motion in the lumbar region, there will be a decrease in the time spent to perform repeated flexion of the trunk. There was also a significant correlation between spinal mobility measures and repeated trunk flexion. Spinal mobility correlated with all the physical performance task Except 5 - minute walk and Sorensen fatique test in the female, although this was not statistically significant. One of the possible reasons for this observation may not be unconnected with the small sample size of females in this study.

It was hypothesized that there would be no significant correlation between pain intensity and functional disability of the subjects. The results of the study showed that there was no significant correlation between pain intensity and functional disability. This implies that changes in pain intensity may not necessarily lead to a considerably change in the physical disability of the subjects. Jensen et al (1992) and Co et al (993) also studied the correlation between pain and back disability using Oswestry Disability Questionnaire in subjects with chronic low back pain and found that there was no significant relationship between pain intensity and functional disability of their subjects. This finding is in agreement with reports of EppingJordan et al (1998), who, in their study on male patients with LBP, reported that pain intensity was not predictive of changes in disability of their subjects. This is however contrary to the findings of Gronblad et al (1997), who reported that both pain intensity and functional disability correlated in male subjects.

\section{CONCLUSION}

It is generally assumed that increase in pain would necessarily result in increase in functional disability thus the assessment of patients' disability is often neglected. Although self report of disability has been shown to correlate moderately with performance tasks (Simmonds et al, 1998), the results of this study showed that clinically relevant improvements in pain may lead to almost unnoticeable change in disability. Therefore, these variables (pain and functional disability) should be assessed separately when evaluating the outcome of the treatment of patients with low back pain. Also, patients' 'selfreports' of disability may not be adequate in making clinical judgement of their condition without the objective assessment of their physical performance. Based on the outcome of 
this study it was recommended that both performance - based and self - report measures of disability should be used in order to obtain a comprehensive picture of the disability of patients with chronic low back pain.

\section{REFERENCES}

- Abenhaim L, Rossignol M, Valat JP, et al. (2000) The role of activity in the therapeutic management of back pain: Report of the International Paris Task Force on Back Pain. Spine. 25 (supplement 4): 1S-33S.

- Andersson GBJ, (1999) Epidemiologic features of chronic low back pain Lancent. 354: 581-585.

- Burton AK, Tillotson KM, Symonds TL, Berk C, Mattheuson T. (1996) Occupational risk factor for the first-onset and subsequent course of low back pain. Spine. 21: 2612-2620.

- Co YY, Eaton S, Maxwell MW. (1993) The relationship between the St Thomas and Oswestry disability scores and the severity of low back pain. Journal of Manipulative and Physiological Therapeutics. 16: 14-18.

- Deyo RA. (1988) Measuring the functional status of patients with low back pain. Archives of Physical Medicine and Rehabilitation. 69: 10441053.

- Deyo RA, Battie M, Beurskens AJ, et al. (1998) Outcome measures for low back pain research: a proposal for standardized use. Spine. 23: 20032013.

- Deyo RA, Weinstein JN. (2001) Low back pain. New England Journal of Medicine. 5 (344): 363370.

- Epping - Jordan JE, Wahlgren DR, Williams RA, et al. (1998) Transition to chronic pain in men with low back pain: predictive relationships among pain intensity, disability, and depressive symptoms. Health Psychology. 17 (5): 421-427.

- Frymoyer JW. (1998) Back pain and sciatica. New England Journal of Medicine. 318: 291300.

- Gronblad M, Hurri H, Kouri JP. (1997) Relationships between spinal mobility, physical performance tests, pain intensity and disability assessments in chronic low back pain patients. Scandinavian Journal of Rehabilitative Medicine, 29 (1):17-24.

- Harding VR, Williams AC, Richardson PH, et al. (1994) The development of a battery of measures for assessing physical functioning of chronic pain patients. Pain. 58: 367-375.

- Moffroid MT, Haugh LD, Henry SM, Short B. (1994) Distinguishable groups of musculoskeletal low back pain patients and asymptomatic control subjects based on physical measures of the NIOSH Low Back Atlas. Spine. 19: 1350-1358.

- Mooney V. (1987) Impairment, disability and handicap. Clinical Orthopaedics. 221: 14-25.

- Nelson A, Nestor M. (1988) Atlas of standardized low-back tests and measures of the National Institute for Occupational Safety and Health. Scandinavian Journal of Work and Environmental Health. 14(suppl 1): 82-84.

- Simmonds MJ, Olson SL, Jones S, et al. (1998) Psychometric characteristics and clinical usefulness of physical performance tests in patients with low back pain. Spine. 23: 24122421.

- Simmonds MJ. (1999) Physical Function and Physical Performance in Patients With Pain: What Are the Measures and What Do They Mean? Seattle, Washington: IASP Scientific Program Committee, IASP Press.

- Turk DC. (1997) The role of demographic and psychosocial factors in transition from acute to chronic pain. In TS Jenson, JA Turner \& S Wiesenfeld-Hallin (Eds.), Proceedings of the eighth world congress on pain, progress, and pain research and management. Seattle: IASP Press. Vol. 8: 185-213.

- Waddell G. (1987) A new clinical model for the treatment of low-back pain. Spine, 12: 632-644.

- Waddell G, Somerville D, Henderson I, Newton M. (1987) Clinical evaluation of physical impairment in chronic low back pain. Spine. 17: 617-628.

- World Health Organization (2001) International Classification of functioning and Disability (ICF). 